\title{
Electrical Characterization of Irradiated Prototype Silicon Pixel Sensors for BTeV
}

\author{
Maria R. Coluccia, J.A. Appel, G. Chiodini, D.C. Christian, S. Cihangir, S.W. Kwan, and G. Sellberg
}

\begin{abstract}
The pixel detector in the BteV experiment at the Tevatron (Fermi Laboratory) is an important detector component for high-resolution tracking and vertex identification. For this task the hybrid pixel detector has to work in a very harsh radiation environment with up to $10^{14}$ minimum ionizing particles/ $\mathrm{cm}^{2} /$ year. Radiation hardness of prototype $\mathbf{n}^{+} / \mathbf{n} / \mathbf{p}^{+}$silicon pixel sensors has been investigated. We present Electrical characterization curves for irradiated prototype $\mathbf{n}^{+} / \mathbf{n} / \mathbf{p}^{+}$sensors, intended for use in the BTeV experiment. We tested pixel sensors from various vendors and with two pixel isolation techniques: p-stop and p-spray. Results are based on irradiation with $200 \mathrm{MeV}$ protons up to $6 \times 1^{14}$ protons $/ \mathrm{cm}^{2}$.
\end{abstract}

\section{INTRODUCTION}

$\mathrm{B}$ $\mathrm{TeV}$ [1] is an experiment expected to run in the new Tevatron $\mathrm{CO}$ interaction region at Fermi National Laboratory (Illinois, USA) in 2008. The main goal of the experiment is to perform precision studies of $b$ and $c$ quark decays, with particular emphasis on mixing, CP violation, rare and forbidden decays [1]. This experiment exploits two important advantages of the "forward" region: the correlation in the direction of the produced $b$ and anti- $b$, that improves the flavor tagging efficiency, and the boots that allows to develop an efficient trigger algorithm based upon the identification of detached charm and beauty decay vertices.

The proposed BTeV spectrometer is shown in Fig. 1. It is a one-arm spectrometer, covering the forward region of the interaction region (IR). The tracking system is designed to reconstruct tracks and measure their angles and momenta over an aperture of $\pm 300 \mathrm{mrad} \times \pm 300 \mathrm{mrad}$.

The key design features of BTeV are constituted by a dipole centered on the interaction region, a precision vertex detector based on planes of pixel detectors placed inside the magnet, a vertex trigger at Level 1[2] and an excellent particle identification. Among these, it is important to underline the unique feature of $\mathrm{BTeV}$ experiment that the vertex detector will be used in the Level-1 trigger to select events with secondary vertices; consequently, the vertex detector must have superior pattern recognition power, small track extrapolation error, and good performance even after

Manuscript received July 16, 2002. This work was supported in part by the U.S. Department of Energy.

All authors are with Fermi National Accelerator Laboratory, P.O. Box 500 Batavia, IL 60510 USA. high radiation dose. Silicon pixel sensors were chosen because they provide very accurate space point information, intrinsically low noise, low occupancy due to large number of channel, better patter recognition capability (compared to strip detectors), high response speed, and high radiation hardness.

We report here on the tolerance of hybrid silicon pixel components intended for use in the $\mathrm{BTeV}$ vertex detector.

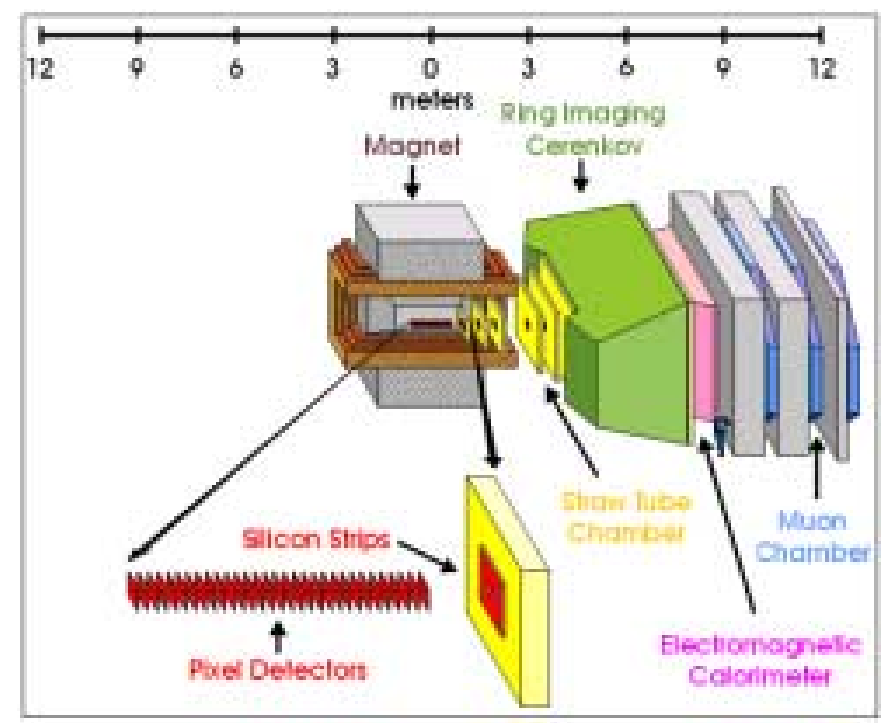

Fig. 1. BTeV detector layout.

\section{BTEV PIXEL SYSTEM}

The BTeV vertex detector is located at the center of a 1.6 $\mathrm{T}$ dipole magnet. It consists of a regular array of 30 "stations" of "planar" pixel detectors, uniformly distributed over $1,28 \mathrm{~m}$ in order to mach the long interaction region of the Tevatron which has a $\sigma_{\mathrm{Z}}$ of $30 \mathrm{~cm}$. Each station contains one plane with the narrow pixel dimension vertical, and one with the narrow dimension horizontal. The unit cell is $50 \mu \mathrm{m}$ $\times 400 \mu \mathrm{m}$. The stations are split so that the sensors can be moved away from the beam while the beams are accelerated and during other unstable beam conditions. See Fig. 2 for details.

The vertex detector contains nearly 22 million rectangular pixels. A dedicated electronics cell reads out each sensor element, and a "bump bond" connects the sensor pixel to the readout cell. The basic building block of the detector is a 
hybrid assembly consisting of a sensor, a number of readout chips (FPIX2 chip [3] developed at Fermilab), and a flexible printed circuit (a high-density interconnect, HDI), which carries I/O signals and power. The sensors are variously sized to accept variable numbers of readout chips to make the required half-plane shape. Each readout chip is "flip-chip" mated to 22 columns of 128 rows of pixels on the sensors, corresponding to 2,816 active channels per readout chip. Each readout chip covers an active area approximately 0.64 $\mathrm{cm} \times 0.92 \mathrm{~cm}$. To avoid any dead space between adjoining readout chips, the pixels on the sensors corresponding to the edge of the readout chip (first and last column) are extended to $600 \mu \mathrm{m}$ length.

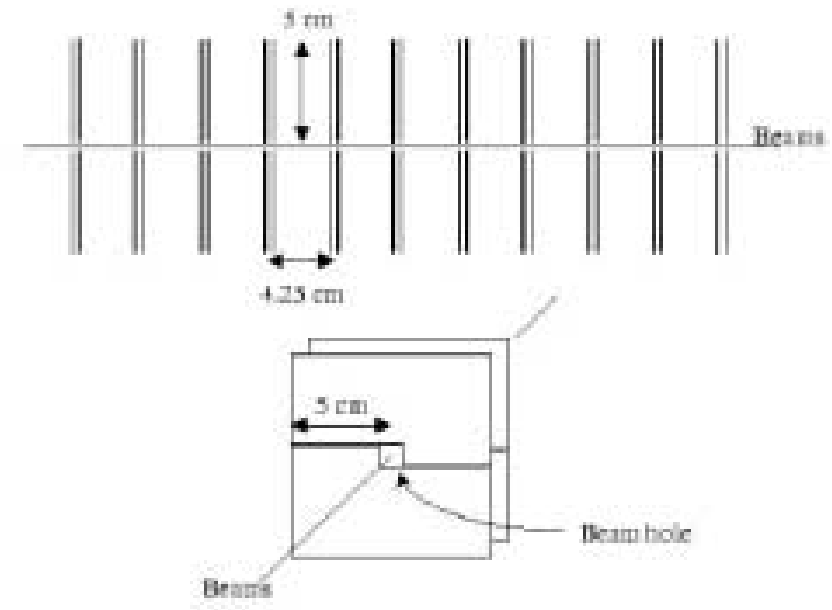

Fig. 2. Schematic drawing of part of the BTeV vertex detector.

Each station has a small square hole of $\pm 6 \mathrm{~mm} \mathrm{x} \pm 6 \mathrm{~mm}$ for the beam to pass through. At such small distance from the colliding beams, the pixel detectors will be exposed to a significant level of irradiation. At the full luminosity at which we plan to operate, the innermost pixel detector will receive an equivalent fluence of $\sim 10^{14}$ minimum ionizing particles $/ \mathrm{cm}^{2} /$ year. This will lead to radiation damage of the silicon pixel sensors.

Due to this harsh environment, the performance of the $\mathrm{BTeV}$ pixel sensors will be degraded with time. The degradation includes an increase of both leakage current and full depletion voltage, and a decrease in charge collection efficiency. These are problems that need to be addressed by all next generation hadron collider experiments. As a result, there is a worldwide effort to address these technical challenges. Solutions include the design of multi guard ring structures to avoid avalanche breakdown along the edge, low resistivity silicon substrates to delay type inversion, and oxygenated wafers to reduce the effects of radiation-induced defects in the silicon lattice.

\section{THE SENSOR CONCEPT}

$\mathrm{BteV}$ is is designed to cover the "forward" region of proton-antiproton interaction point at a luminosity of $2 \times 10^{32}$ $\mathrm{p} / \mathrm{cm}^{2} / \mathrm{s}$. Because of the hard radiation environment the pixel detector requirements are extremely challenging. All components have to be radiation hard up to a fluence of $10^{14}$ particles $/ \mathrm{cm}^{2} /$ year in the most critical region at $6 \mathrm{~mm}$ from the beam.

The ATLAS pixel collaboration successfully developed for LHC a radiation hard sensor [4], which is expected to meet also the requirement for $\mathrm{BTeV}$ pixel detector. The design of our silicon sensors is guided by the necessity to operate the device at a few hundred volts without the risk of junction breakdown or micro-discharge. For this a multiple guard ring structure is used to control the potential drop toward the cut edge on the $\mathrm{p}$-side. These structures maintain the $\mathrm{p}$ edges of the sensors at the same potential as the $\mathrm{n}^{+}$-side, which sits at the input potential of the readout-chip. Finally, the hardening of the silicon itself is following the ROSE collaboration results, which developed the DOFZ type low resistivity silicon with increased oxygen by diffusion [5]. Our design is taking advantage of all these previous results.

$\mathrm{BTeV}$ pixel sensors have $\mathrm{n}^{+} / \mathrm{n} / \mathrm{p}^{+}$configuration in order to operate in condition of partial depletion and, therefore, to increase the lifetime of the silicon sensors. After bulk type inversion, the depleted region grows from the $\mathrm{n}^{+}$side of the junction and the sensor can operate partially depleted. Such operation might be necessary if the full depletion voltage becomes excessively large after substrate type inversion caused by the high irradiation. However, for $\mathrm{n}^{+} / \mathrm{n}$ devices, it is necessary to provide explicit electrical isolation between neighboring $\mathrm{n}^{+}$electrodes. Without isolation, the accumulation layer induced by oxide charge buildup would short the individual $\mathrm{n}^{+}$electrodes. Various isolation techniques have been developed for silicon pixel sensors. Here we explore two techniques: $p$-stop and moderated pspray.
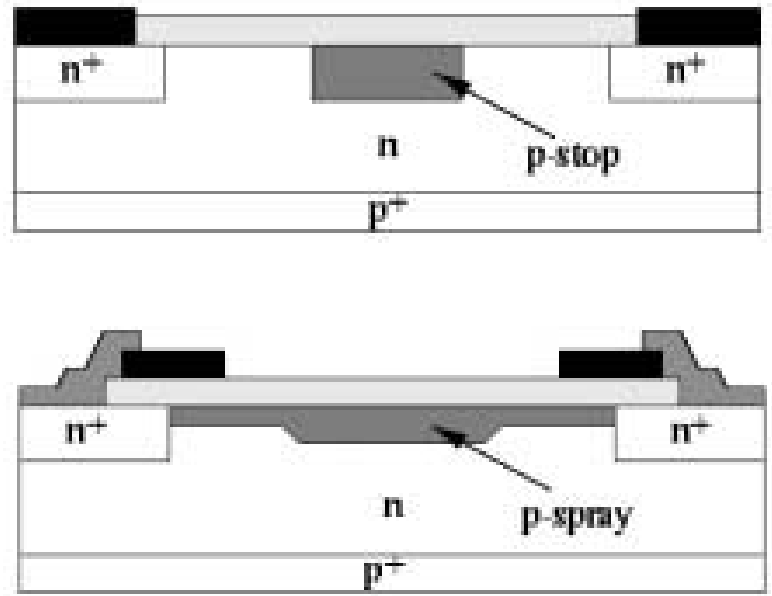

Fig. 3. Schematic views of the implants in the p-stop (top) and moderated p-spray (bottom) electrode isolation methods.

In the case of the p-stop isolation technique, a high dose pimplant surrounds each $\mathrm{n}^{+}$-type region. We tested pixel sensors having two different layouts of p-stop [6] electrode 
isolation: individual and common p-stops. For the individual p-stop there is a p-implant ring (atoll) around each pixel. For the common $\mathrm{p}$-stop, there is a continuous p-implant between pixel columns and rows. The p-spray [7] isolation technique was developed by the ATLAS collaboration and consists of a medium dose p-implant that is applied to the entire n-side and is overcompensated by the high dose $\mathrm{n}^{+}$pixel implants. To increase radiation hardness and also to increase the breakdown voltage before irradiation, a "grading" of p-spray implantation (moderated p-spray) [8] is used. This technique leads to a step in the effective p-spray dose along the gap between two $\mathrm{n}^{+}$-implants. In the middle of the gap, the normal p-spray dose is reached. This guarantees the inter-pixel isolation. Near the edges, the p-spray dose is lower in order to minimize the electric field strength in these regions and therefore improve breakdown performance [9]. Fig. 3 shows a schematic view of both p-stop and p-spay isolation methods.

In the p-spray technique, a bias grid structure was implemented in order to test the sensors under full bias before assembly. This structure is very important for quality control during mass production and is very useful for testing the sensors before and after irradiation in the development phase. Moreover, in case of missing bonds, this bias grid acts as a safety feature during operation, maintaining the unconnected $\mathrm{n}^{+}$electrode potentials closed to ground. The bias grid connects every pixel via an equally sized punchthrough gap applying the same potential to every individual pixel. For the p-stop design it is not possible to have a bias grid because this would have to cross the p-stop implant.

\section{Devices Under Test}

We have tested sensors from three vendors: the p-stop sensors are from SINTEF Electronics and Cybernetics (Oslo, Norway), the p-spray sensors are from TESLA (Roznov, Czech Republic) and from CiS (Erfurt, Germany). The base material for the p-stop sensors is low resistivity (1.0-1.5 $\mathrm{k} \Omega$ $\mathrm{cm})<100>$ silicon, $270 \mu \mathrm{m}$ thick. For the p-spray sensors were fabricated using higher resistivity $(2-5 \mathrm{k} \Omega \mathrm{cm})<111>$ silicon, $250 \mu \mathrm{m}$ thick. Some of the SINTEF and CiS wafers and all the TESLA wafers have been oxygenated.

We tested three different pixel array sizes for p-stop sensors and one for the $\mathrm{p}$-spray. The first $\mathrm{p}$-stop array (called "test-sized sensor") contains 12 × 92 cells and all these cells, except for four, are connected together. This structure was designed to study the behavior of a single cell. The second array (called "FPIX0-sized sensors") contains $12 \times 64$ cells and it is designed to be read out by a single FPIX0 chip [10], that is the very first readout implementation for BTeV. The third array (called "FPIX1-sized sensors"), both for p-stop and p-spray, contains $18 \times 160$ cells and it is designed to be read out by a single FPIX1 chip [11] that is the first complete high speed digital pixel readout architecture implementation before the development of the final FPIX2 chip. As mentioned above for the p-spray arrays, a bias grid is implemented. In the p-stop array, there is no bias grid and unlike the test-sized sensors, in this case no cells are connected together.

We have four different guard ring structures on the tested devices. Information on the guard ring structures and tests can be found in our previous paper [12].

\section{EXPERIMENTAL PROCEDURES}

Electrical characterization of the devices was performed with standard techniques ( $\mathrm{I}-\mathrm{V}, \mathrm{V}_{\mathrm{g}-\text {-ring }}-\mathrm{V}$ and $\mathrm{C}-\mathrm{V}$ curves) before and after irradiation. We used a Keithley 237 as power supply and current monitor, and both QTech 7600 meter for the $\mathrm{C}-\mathrm{V}$ measurements.

All the measurements were performed using a probe station placed in a dark box in a clean room. Continuous monitoring of temperature and humidity were performed, and all the measurements reported were done at $0 \%$ relative humidity, achieved by flowing dry nitrogen in the dark box. In order to investigate the stability of the electrical characteristics, several measurements were performed in various humidity conditions (ranging from $0 \%$ to $40 \%$ ), but no significant difference was detected.

The measurements were performed with the p-side (sensor back-plane) biased through one probe, the guard ring floating and the n-side grounded through the support chuck.

We measured the leakage current and the capacitance for the whole sensor without considering any correction for the contribution from the guard ring region. We performed some measurements before irradiation in which the innermost guard ring was biased to the same potential as the p-side. However, we found that the contribution from the guard ring region was negligible before irradiation.

Several devices were characterized before irradiation and some of these also after irradiation. The proton irradiation took place at the Indiana University Cyclotron Facility (IUCF) with a $200 \mathrm{MeV}$ proton beam. The displacement damage cross section for $200 \mathrm{MeV}$ protons $(90.5 \mathrm{MeVmb})$ [13] is almost exactly the same as the value conventionally assigned to $1 \mathrm{MeV}$ neutrons (95 MeV-mb) [14] so we quote our results as a function of proton fluence rather than $1 \mathrm{MeV}$ neutron equivalent fluence. The beam profile was measured by exposing a sensitive film. The beam spot, defined by the circular area where the flux is within $90 \%$ of the central value, had a diameter of $1.5 \mathrm{~cm}$, comfortably larger than the sensor size (the FPIX1-sized sensor is $\sim 1 \mathrm{~cm} \mathrm{x} 1 \mathrm{~cm}$ ). Before the exposure, the absolute fluence was measured with a Faraday cup; during the exposure the relative fluence was determined with a Secondary Electron Emission Monitor.

We used a PC board with a big opening in the middle (4 inch $\times 4$ inch) where we placed the sensors with simple cardboard supports. The irradiation was done in air at room temperature and took no more than six hours. The exposures with multiple boards were done placing the boards about 2 $\mathrm{cm}$ behind each other and with the pixel side facing the beam. Mechanically, the boards were kept in position by an open 
aluminum frame. A maximum of six boards were exposed each time, and therefore the beam energy degradation was negligible. After irradiation, the tested devices were kept at minus $15{ }^{\circ} \mathrm{C}$ in order to suppress any beneficial and/or reverse annealing process.

The measurements after irradiation were performed in a condition in which the plateau of the beneficial annealing has not been reached. We are interested in investigating the behavior of the sensors in an environment that is as close as possible to the real experiment. The operational temperature of the vertex detector in $\mathrm{BTeV}$ will be between $-5^{\circ} \mathrm{C}$ and $10{ }^{\circ} \mathrm{C}$ and therefore the pixel sensors will not profit from beneficial annealing. For this reason, we decided to store the irradiated sensors at low temperature just after irradiation. The measurements at room temperature took no more than a few hours.

Typically, measurements were made thirty days after irradiation.

\section{Result AND Discussion}

\section{A. P-Stop Sensor Performance}

We tested several p-stop sensors before and after irradiation. Fig. 3 shows the typical I-V curves before irradiation for a test-sized sensor, and the I-V curves for a FPIX0-sized sensors with indium bumps deposited on the $\mathrm{n}^{+}$ side that were then glued with conducting silver epoxy to a PC board, and for a FPIX1-sized sensors bonded to a FPIX1 readout chip.

The test-sized sensors have very good performance, high breakdown voltage $(>500 \mathrm{~V})$ and small leakage current $(\sim 10$ $\mathrm{nA} / \mathrm{cm}^{2}$ after full depletion). For the FPIX0-sized and FPIX1sized sensors the tests performed on the bare sensors show that, although the current is also small, the breakdown voltage is lower (typically just above $300 \mathrm{~V}$ ). The same results were found for all the sensors that were tested.

As we reported in a previous paper [15], the changing in the breakdown voltage performance for the FPIX0-sized and FPIX1-sized sensors is likely due the fact that we can not bias properly all the cells on the bare sensors. In [15] we report the result obtained considering FPIX1-sized sensors bump bonded to a readout chip and FPIX1-sized sensors with indium bumps deposited on the $\mathrm{n}^{+}$side that were glued with conducting silver epoxy to a piece of silicon in order to mimic the presence of the readout chip. The results obtained for these sensors were very promising, in fact the breakdown voltage performances improve and are similar to the one obtained for the test-sized sensors. Here we report the results obtained for irradiating the FPIX1-sized sensors glued to the silicon in order to be sure that all the cells are properly biased.

The same problem rise for the FPIX0-sensors, we'll discuss later in this section why we glued the sensors to a PC board.

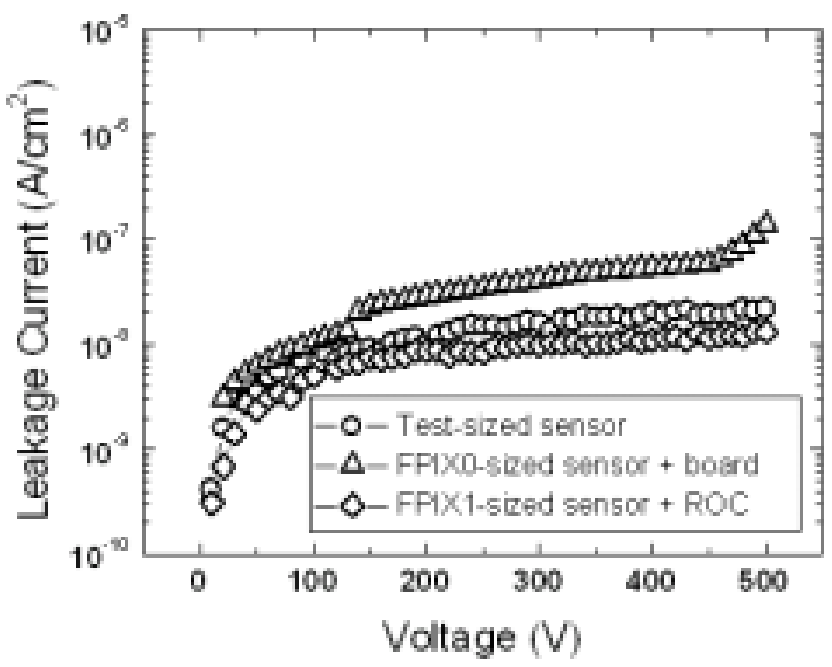

Fig. 3. I-V characteristics for un-irradiated p-stop pixel sensors.

Several test-sized and FPIX1-sized sensors glued to a piece of silicon were irradiated up to $6 \times 10^{14} \mathrm{p} / \mathrm{cm}^{2}$. Fig. 4 shows the IV characteristics for an FPIX1-sized sensor irradiated up to $3 \times 10^{14} \mathrm{p} / \mathrm{cm}^{2}$. The leakage current after irradiation increased by several orders of magnitude. However, operating at lower temperature can significantly reduce this leakage current. The measurements shown in Fig. 4 were done at $23{ }^{\circ} \mathrm{C}$. We investigated the variation of the leakage current with the temperature in previous work and, as expected, we observed that the current decreases exponentially with temperature [12]. Up to $6 \times 10^{14} \mathrm{p} / \mathrm{cm}^{2}$, the sensors have a breakdown voltage higher than $500 \mathrm{~V}$.

As expected, the leakage current after irradiation has a nearly linear dependence on fluence. In fact, the increase of the leakage current $\Delta \mathrm{I}$ (i.e. the difference between the currents measured after and before irradiation) shows a linear dependence on the fluence: $\Delta \mathrm{I}=\alpha \Phi \mathrm{V}$ where $\alpha$ is the damage constant, $\Phi$ is the fluence, and V is the volume. Fig. 5 shows the fluence dependence of the increase in leakage current normalized to volume. We obtained a value for the leakage current damage constant $\alpha$ of $(4.98 \pm 0.0051) \times 10^{-17}$ $\mathrm{A} / \mathrm{cm}$. This is comparable to previous measurements [12]. However, it must be noted that these various measurements were taken under a wide variety of conditions.

In order to investigate the bulk damage induced by radiation, we measured the dependence of the deletion voltage (and therefore the operating voltage) on the proton fluence. It is well known that the depletion voltage depends on the effective doping concentration, which changes with the irradiation [16]. The depletion voltage is normally defined as the bias voltage that one needs to apply to the sensors in order to have the semiconductor bulk completely depleted of free carriers. We extract this value from $\mathrm{C}-\mathrm{V}$ curves as the intersection point of two fitted straight lines in the $\log C-\log \mathrm{V}$ plot. Fig. 6 shows the dependence of the full depletion voltage on proton fluence for the p-stop sensors. We see that the full depletion voltage at $4 \times 10^{14} \mathrm{p} / \mathrm{cm}^{2}$ is still very low, lower than the value before irradiation $(\sim 210 \mathrm{~V})$. 
This characteristic is due to the low resistivity of the silicon. Even up to a fluence of $6 \times 10^{14} \mathrm{p} / \mathrm{cm}^{2}$ (equivalent to 6 years of $\mathrm{BTeV}$ running at the nominal luminosity of $\left.2 \times 10^{32} \mathrm{~cm}^{-2} \mathrm{sec}^{-1}\right)$, the depletion voltage is still low compared to the breakdown voltage $(>500 \mathrm{~V})$.

More results for the p-stop SINTEF sensors can be found in our previous work [12].

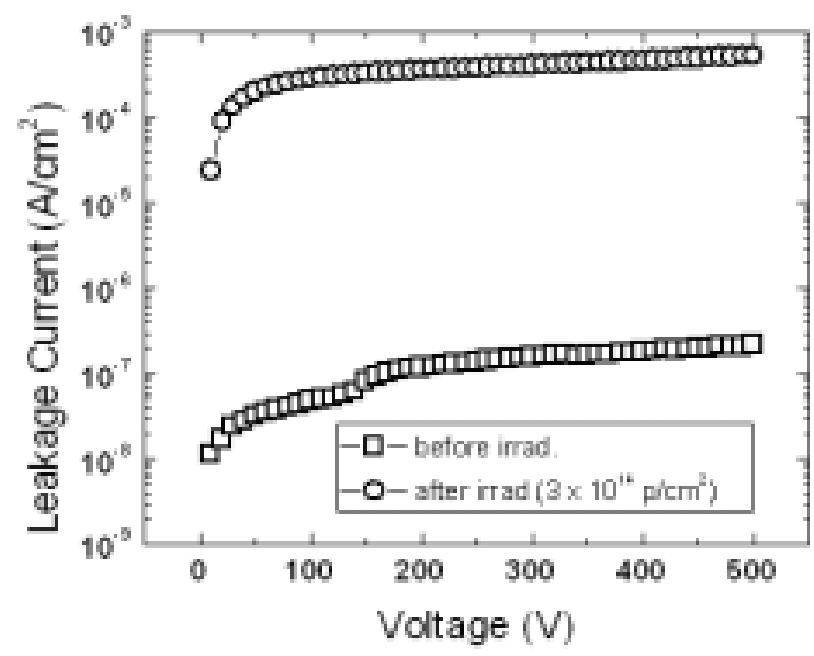

Fig. 4. I-V curves for a common p-stop FPIX1-sized sensor glued to a piece of silicon before and after irradiation up to $3 \times 10^{14} \mathrm{p} / \mathrm{cm}^{2}$. The measurements were performed at room temperature.

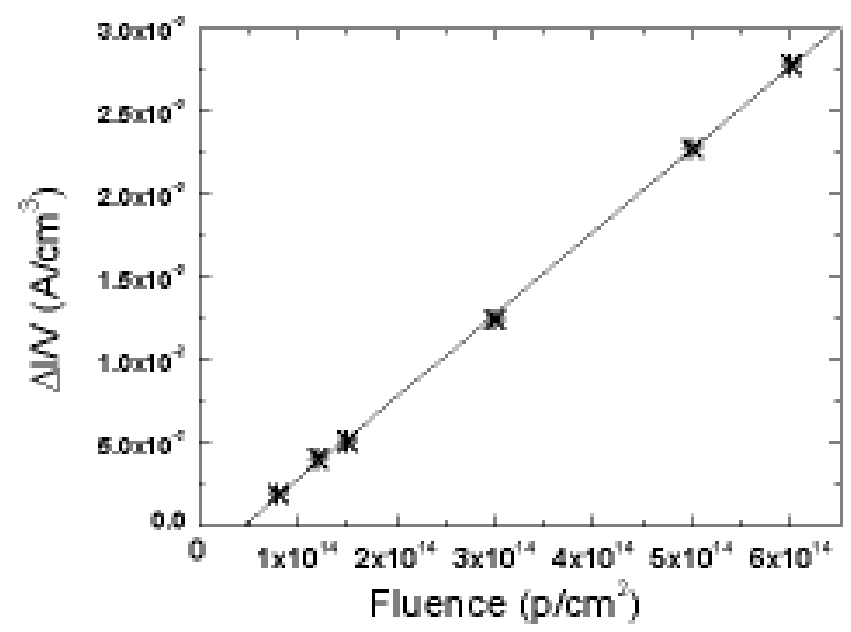

Fig. 5. Fluence dependence of the increase in leakage current for p-stop sensors. The current was measured at room temperature $\left(23^{\circ} \mathrm{C}\right)$.

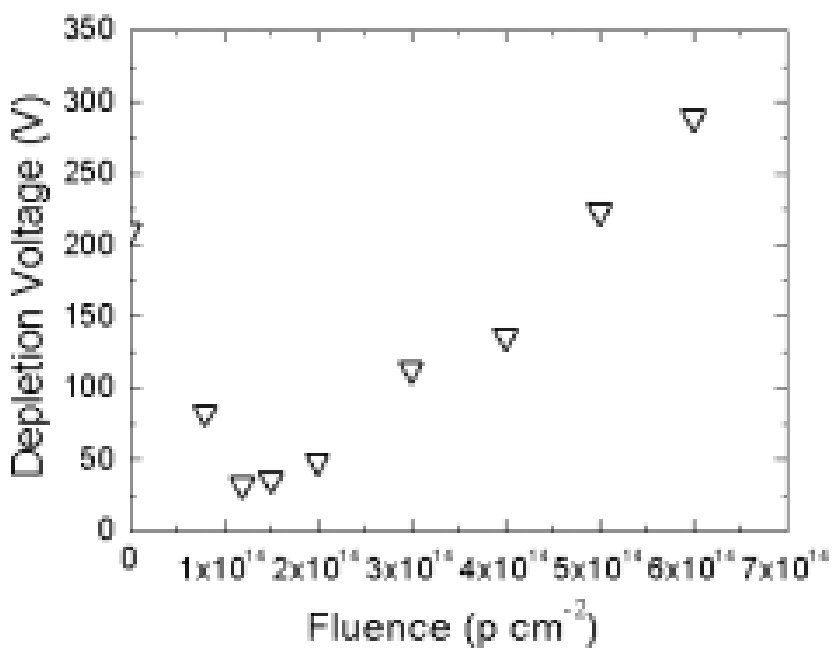

Fig. 6. Depletion voltage as function of proton fluences for p-stop pixel sensors.
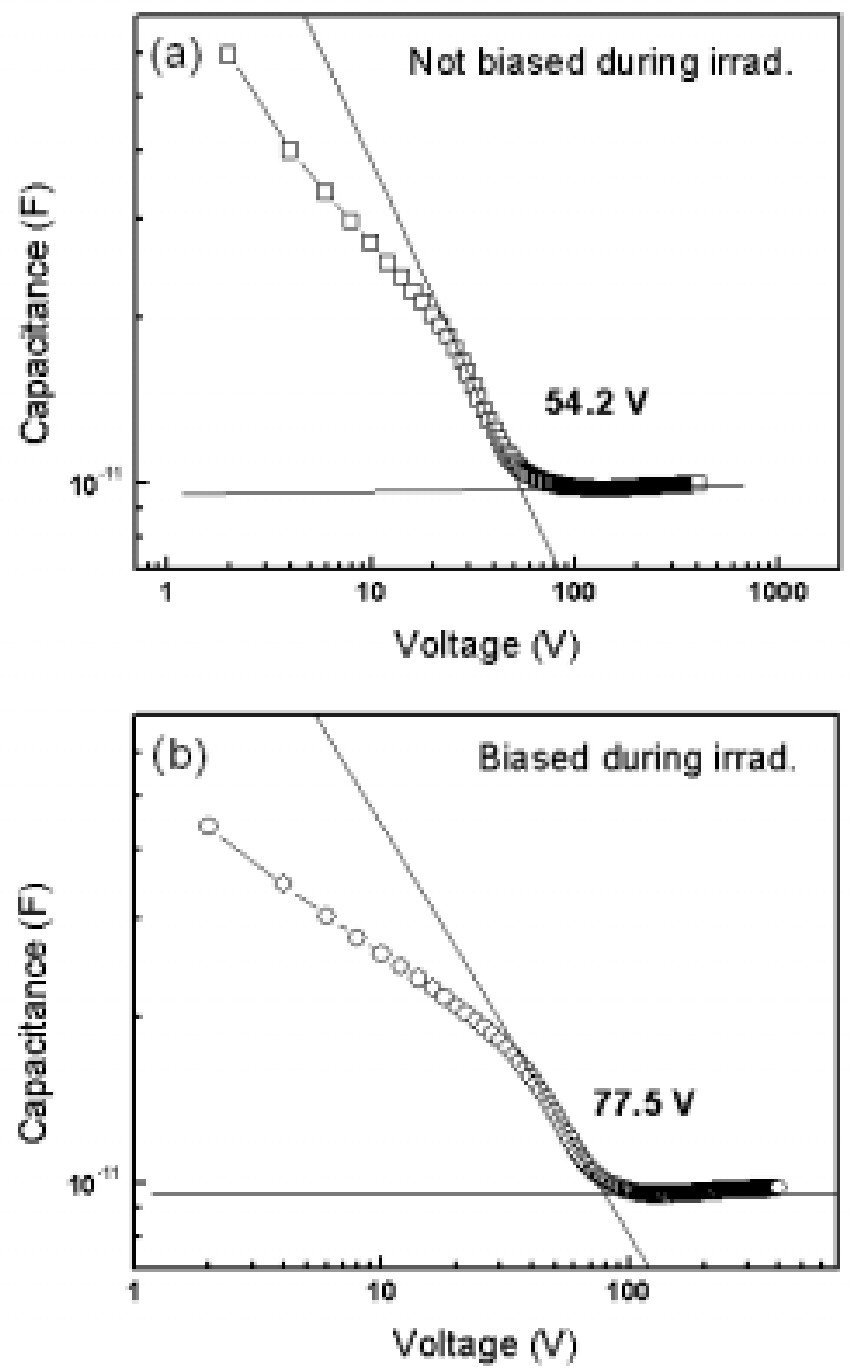

Fig. 7. C-V curves for an FPIX0-sized p-stop not biased during irradiation (a) and biased during (b). 
We also irradiated two FPIX0-sized glued to a PC board. As we mention in the introduction section, the change of effective impurity concentration in silicon, caused by irradiation with fast heavy particles, results in a change of the full depletion voltage. Typically, the full depletion voltage is obtained from $\mathrm{C}-\mathrm{V}$ measurements. All the sensors that we tested up to now were irradiated without bias. In the real experiments, however, detectors have to be under bias during data taking. Therefore, we have set up a first test to study the effect of the bias on changes of full depletion voltage induced by proton irradiation.

We used two FPIX0-sized sensors glued with conductive silver epoxy to a PC board. They were irradiated up to $1.5 \times$ $10^{14} \mathrm{p} / \mathrm{cm}^{2}$ and one of them was biased during and after irradiation. Like for all the other sensors, also these devices were kept at minus $15^{\circ} \mathrm{C}$ after irradiation.

Fig .7 shows the $\mathrm{C}-\mathrm{V}$ curves for these two sensors. We also calculated the depletion voltage as the intersection point of two fitted straight lines. As we can see there is difference of more than $20 \mathrm{~V}$ between the two sensors. More investigations are necessary in order to better understand the impact of this changing in the depletion voltage, and therefore in the effective impurity concentration, on the $\mathrm{BTeV}$ experiment. More tests are scheduled for the next months in order to arrive at more reliable prediction of the influence of bias on the depletion voltage and therefore on the operating voltage..

\section{B. P-Spray Sensor Performance}

Several wafers from CiS and two from TESLA were tested. Apart from a few sensors that show higher leakage current and low breakdown voltage $(<300 \mathrm{~V})$, the typical I-V curves for FPIX1-sized p-spray sensors show a breakdown voltage higher than $500 \mathrm{~V}$ and a low leakage current (the breakdown voltage is defined as the voltage for which the current increases steeply and is bigger than $1 \mu \mathrm{A}$ ).

We have irradiated only two of these sensors in two different steps: one was irradiated up to $8 \times 10^{13} \mathrm{p} / \mathrm{cm}^{2}$ the first time and up $2.3 \times 10^{14} \mathrm{p} / \mathrm{cm}^{2}$ the second time, and the other up to $1.2 \times 10^{14} \mathrm{p} / \mathrm{cm}^{2}$ the first time and up to $4.2 \times 10^{14}$ $\mathrm{p} / \mathrm{cm}^{2}$ the second time. Fig. 8 shows the increase in the leakage current due to irradiation for the sensor irradiated up to $2.3 \times 10^{14} \mathrm{p} / \mathrm{cm}^{2}$. The current increased several orders of magnitude, as was the case for the p-stop sensors that we tested. From Fig. 8 shows also another import characteristic of the p-spray isolation technique, that is that the breakdown voltage performance increases after irradiation [7]. In fact, in this case, the electric filed between the doped isolation layer and the adjacent $\mathrm{n}^{+}$cell decreases with the increasing charge oxide due to the irradiation. This fact is due to the compensation of the acceptor doping by the oxide charge.

Fig. 9 shows the fluence dependence of the increase in leakage current normalized to volume for the p-spray sensors. We obtained a value for the leakage current damage constant $\alpha$ of $(4.98 \pm 0.0040) \times 10^{-17} \mathrm{~A} / \mathrm{cm}$. This value is comparable with the one that we obtained for the p-stop sensor.

Also for these sensors we investigated the dependence of the full depletion voltage on proton fluence (see Fig. 10) and again we found that up to $4.2 \times 10^{14} \mathrm{p} / \mathrm{cm}^{2}$ the depletion voltage is still very low compared with the breakdown voltage $(>500 \mathrm{~V})$.

From a comparison between Fig. 6 and Fig. 10 we can see that the type inversion occurs at a lower dose for the highresistivity p-spray sensors than for the low-resistivity p-stop sensors.

More tests are in progress in order to better investigate the performance of the p-spray isolation technique. We received recently several wafers from TESLA in which the $\mathrm{BTeV}$ layout is implemented. These are low resistivity moderated $\mathrm{p}-$ spray sensors.

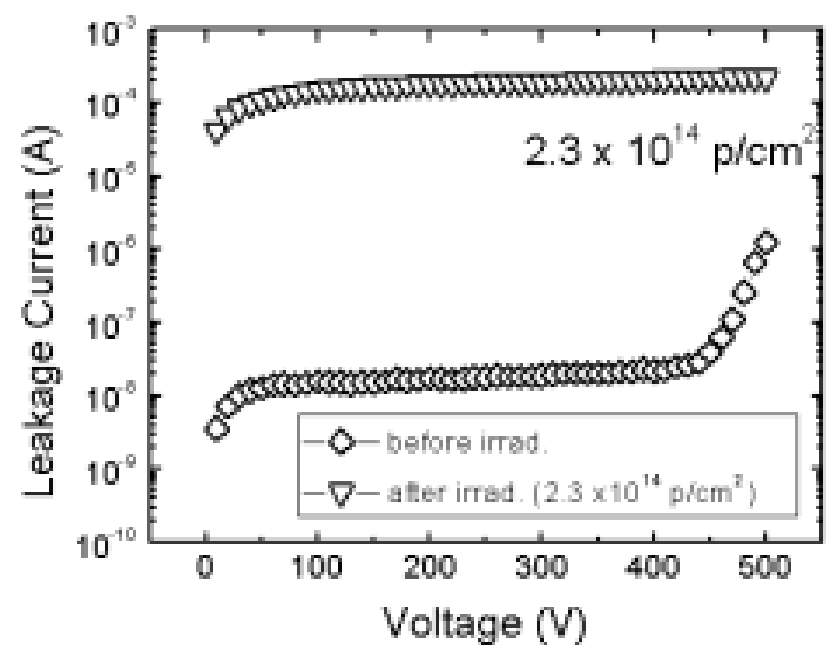

Fig. 8. I-V curves for a FPIX1-sized p-spray sensor before and after irradiation up to $1.2 \times 10^{14} \mathrm{p} / \mathrm{cm}^{2}$. The measurements were performed at room temperature.

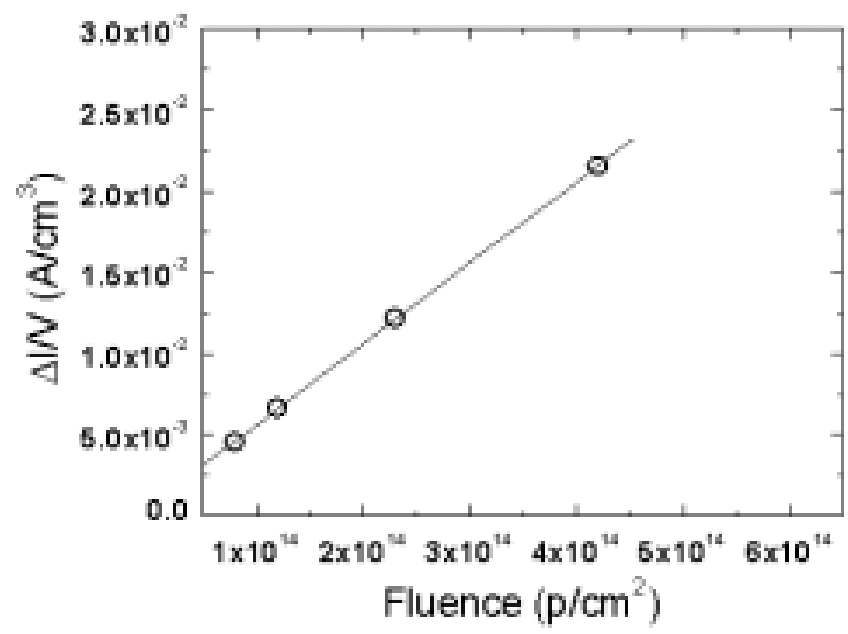

Fig. 9. Fluence dependence of the increase in leakage current for p-spray sensors. The current was measured at room temperature $\left(23^{\circ} \mathrm{C}\right)$. 


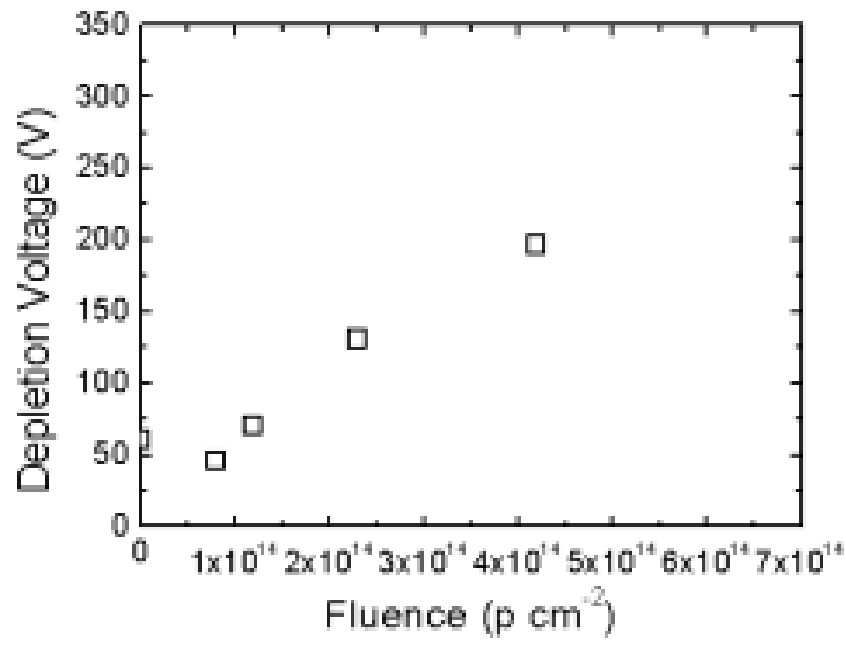

Fig. 10. Depletion voltage as function of proton fluences for p-spray pixel sensors.

\section{CONCLUSION}

A first characterization of the prototype $\mathrm{BTeV}$ silicon pixel sensors is presented. Two different pixel isolation techniques were considered: $\mathrm{p}$-stop and p-spray. For the SINTEF p-stop pixel sensors, experimental results based on I-V and C-V measurements are promising. Most of the tested sensors meet the specifications: leakage current less than $50 \mathrm{nA} / \mathrm{cm}^{2}$ and breakdown voltage above $300 \mathrm{~V}$. From the point of view of the radiation hardness with proton fluence, SINTEF lowresistivity sensors have excellent performance. However, we see that for this pixel layout there is the problem with determining the breakdown voltage in wafer probing. This may imply problems with quality control and yield determination during large scale sensors production.

For the p-spray pixel sensors, the results are promising too. In this case, there are no ground problems due to the fact that a bias grid structure can be implemented. More tests will be performed on low resistivity sensors.

We started to investigate the dependence of the depletion voltage, and therefore of the effective impurity concentration, on the biasing during irradiation. In our first test we fond that biasing a sensor during irradiation leads to a difference respect to the unbiased sensors of about $20 \mathrm{~V}$. More test are scheduled to better understand this problem in the $\mathrm{BTeV}$ experiment context.

We will study the performance of both these detectors in a test beam.

\section{ACKNOWLEDGMENTS}

We would like to thank to the ATLAS collaboration to provide us with $\mathrm{p}$-spray sensors.

\section{REFERENCES}

[1] A. Kulyavtsev, M. Procario, J. Russ, J. You, J. Cumalat, J. Appel, et al.. "BTeV proposal," Fermilab, May 2000.

[2] E. E. Gottschalk, "BTeV detached vertex trigger," Fermilab-CONF-01088-E, June 2001.

[3] R. Yarema, W. Wester and S. Zimmermann, "FPIX2: a radiation-hard pixel readout chip for BTeV," Nucl. Instr. Meth., A 473, 152 (2001).

[4] F. Hugging, et al., "Prototype performance and design of the ATLAS pixel sensors," Elsevier Preprint, 4 Oct 2000.

[5] The ROSE Collaboartion , "3 ${ }^{\text {rd }}$ RD48 Status Report," CERN/LHCC 2000-009.

[6] G. Batagnani, F. Bosi, L. Bosisio, A. Conti, E. Focardi, F. Forti, et al., "Double-sided readout silicon strip detectors for the ALEPH minivertex," Nucl. Instr. Meth., vol. A 277, pp 147-153, 1989.

[7] R. H. Richter, L. Andricek, T. Gebhart, D. Hauff, J. Kemmer, G. Lutz, et al., "Strip detector design for ATLAS and HERA-B using twodimensional device simulation," Nucl. Instr. Meth., vol. A 377, pp 412421, 1996.

[8] G. Lutz, et al., Streifendetektor, Patentoffenlegungsschrift-OS 19620 081 Al 21.11.97, Munich, 1997.

[9] R. H. Richter, et al., "Moderate p-spray-an n-side isolation technique for highly irradiated silicon detectors," Nucl. Instr. Meth., submitted.

[10] A. Mekkaoui, Fermilab-Conf-98/336, 1998.

[11] D. C. Christian, J. A. Appel, G. Cancelo, S. W. Kwan, J. Hoff, A. Mekkaoui, et. Al, "Development of a pixel read out chip for BTeV," Nucl. Instr. Meth., A 335, 144 (1999).

[12] M.R. Coluccia, J.A.Apple, G. Chiodini, D.C. Christian and S.W. Kwan, "Characterization of prototype $\mathrm{BTeV}$ silicon pixel sensors before and after irradiation," IEEE Trans. Nucl.. Sci, vol. 49, no. 4, 2002.

[13] G.P. Summer, E.A. Burke, P. Shapiro, S.R. Messenger, and R.J. Walters, "Damage correlation in semiconductors exposed to gamma, electron, and proton irradiation," IEEE Trans. Nucl. Sci. vol. 40, p 1372, 1993.

[14] Annual Book of ASTM Standard, "Standard practice for characterizing neutron energy fluence spectra in terms of an equivalent monoenergetic neutron fluence for radiation-hardness testing of electronics," E772-94 vol. 12.02 .

[15] M.R. Coluccia, J.A.Apple, G. Chiodini, D.C. Christian and S.W. Kwan, G.Sellberg, and L.Uplegger, "IV and CV curves for irradiated Prototype BTeV Silicon Pixel Sensors," accepted for publication in IEEE Trans. Nucl.. Sci, Dec 2002.

[16] G. Lutz, "Semiconductor radiation detectors: device physics," Springer 1999. 